\title{
Correction to: In utero exposure to gestational diabetes and adiposity: does breastfeeding make a difference?
}

\author{
L. L. Hui ${ }^{1,2}$ - A. M. Li $i^{1}$ E. A. S. Nelson $\mathbb{1}^{1} \cdot$ G. M. Leung ${ }^{2}$ S. L. Lee ${ }^{3}$ - C. M. Schooling $\mathbb{(}^{2,4}$
}

Published online: 2 May 2019

(c) Macmillan Publishers Limited, part of Springer Nature 2019

\section{Correction to: International Journal of Obesity https://doi.org/10.1038/s41366-018-0077-2 published online 17 May 2018}

In the original version of this article, the Publisher incorrectly listed the affiliation of the author, G.M. Leung. The correct affiliation for this author should be: School of Public Health, Li Ka Shing Faculty of Medicine, The University of Hong Kong, Hong Kong SAR, China.

This has now been corrected in both the PDF and HTML versions of the article.

C. M. Schooling

cms1@hku.hk

1 Department of Paediatrics, Faculty of Medicine, The Chinese University of Hong Kong, Hong Kong SAR, China

2 School of Public Health, Li Ka Shing Faculty of Medicine, The University of Hong Kong, Hong Kong SAR, China

3 Department of Paediatrics \& Adolescent Medicine, Li Ka Shing Faculty of Medicine, The University of Hong Kong, Hong Kong SAR, China

4 CUNY Graduate School of Public Health and Health Policy, New York, NY, USA 